\title{
3D Nonholonomic Source Seeking Without Position Measurement
}

\author{
Jennie Cochran, Nima Ghods and Miroslav Krstic
}

\begin{abstract}
We consider the three dimensional problem of directing a nonholonomic vehicle to seek the source of a scalar signal without the use of position information. If we assume the signal strength decays with distance from the source then we achieve convergence to the source by making use of the extremum seeking method. In the kinematic vehicle model we employ, the forward velocity is constrained to a constant and the control inputs are the yaw and pitch velocities. We present a control scheme which tunes these angular velocities and prove the local exponential convergence of this scheme. We also provide simulations which illustrate the behavior of the vehicle under different scenarios, such as static and moving sources, signal fields with spherical and elliptical level sets and parameter regimes not covered by theory.
\end{abstract}

\section{INTRODUCTION}

The study of autonomous vehicle navigation in environments where GPS is unavailable or inertial navigation is too costly is an area of rapidly growing interest. Previously we applied extremum seeking, a non-model based gradient estimation method, to 2D vehicles modeled as unicycles towards the aim of seeking the source of a scalar signal without the use of position information. We considered both a unicycle with a constant forward velocity and a controlled angular velocity [1] and a unicycle with a constant angular velocity and a tuned forward velocity [2]. In each case we proved local convergence to the source. In this paper we extend these results to an underactuated $3 \mathrm{D}$ vehicle.

As before, we consider a vehicle that is capable of sensing a scalar signal emanating from a target source, yet is denied position information. The signal could be many things, including the concentration of a chemical or biological agent, or an electromagnetic, acoustic, thermal or radar signal. The control law for the vehicle relies solely on the signal reading, whose strength decays away from the source, to guide the vehicle towards the source. We employ extremum seeking in the control law, which allows the controller to jointly estimate the gradient and drive the vehicle in the correct direction. However, unlike previous scenarios, in this paper we consider an underactuated vehicle operating in three dimensions instead of two. The extension to three dimensions is interesting because of the choice of vehicle model to consider and because of the type of vehicle movement which might be possible to actuate. We have chosen to address a vehicle with a constant forward velocity and controllable yaw and pitch velocities. We present a local stability proof for the scheme applied to a static source and simulation results.

This work was supported by an NDSEG fellowship, NSF and LANL.

The authors are with the Department of Mechanical and Aerospace Engineering, University of California, San Diego, La Jolla, CA 92093, USA jcochraneucsd.edu
While other groups have considered source seeking problems, [3] and [4], this work is different in that the vehicle has no knowledge of its position or the position of the source, there is no communication between it and other entities, and it has nonholonomic dynamics. Some groups have also considered vehicle movement in three dimensional space, including [5], [6], while others have also used the extremum seeking method in their work outside of the field of autonomous vehicles, including [7], [8], [9], [10], [11], [12], [13], [14], [15] and [16].

We start the paper with a description of the extremum seeking method in Section II and the vehicle model in III. We continue in Section IV with a statement of the stability achieved and prove the local exponential convergence of the vehicle to an "orbit-like" attractor around the source. Section $\mathrm{V}$ illustrates the behavior of the vehicle driven by our control law through simulations. We conclude in Section VI.

\section{OVERVIEW OF EXTREMUM SEEKING}

Extremum seeking is a non-model based gradient estimation method. It employs a periodic forcing of the plant, in this case an autonomous vehicle, to do this. As applied to source seeking, the method works under the assumption that the target creates some signal field that the vehicle can sense. Though the shape of the field is unknown, the strength of the signal decays with distance away from the source and the maximum occurs at the target itself. Only the reading of the signal field is used as input to the extremum seeking controller - position information is not used.

We assume the signal field is distributed according to some unknown nonlinear map $J=f\left(r_{s}\left(x_{s}, y_{s}, z_{s}\right)\right)$ which has an isolated local maximum $f^{*}=f\left(r^{*}\right)$ where $r^{*}$ is the three dimensional location vector of the local maximum. If both the shape of $f$ and position $r_{c}$ of the vehicle were known, and if the vehicle were fully actuated in all three directions, then we could design a control law to force the vehicle's trajectory to evolve according to the gradient dynamical system $\dot{r}_{c}=$ $-\nabla f$. In that case the trajectory of $r_{c}$ would asymptotically converge to the set of stationary points of $f$ where $\nabla f\left(r^{*}\right)=$ 0 . As we know neither the shape of $f$ or the position $r_{c}$ of the vehicle, we employ extremum seeking as a non-model-based estimation method.

As the vehicle is constrained, the method succeeds in simultaneously solving a nonholonomic steering problem and an adaptive optimization problem. Each tuned velocity of an autonomous vehicle (forward or angular) is of the basic extremum seeking form

$$
a_{i} \omega_{i} \cos \left(\omega_{i} t\right)+c_{i} \frac{s}{s+h}[J] \sin \left(\omega_{i} t\right)
$$




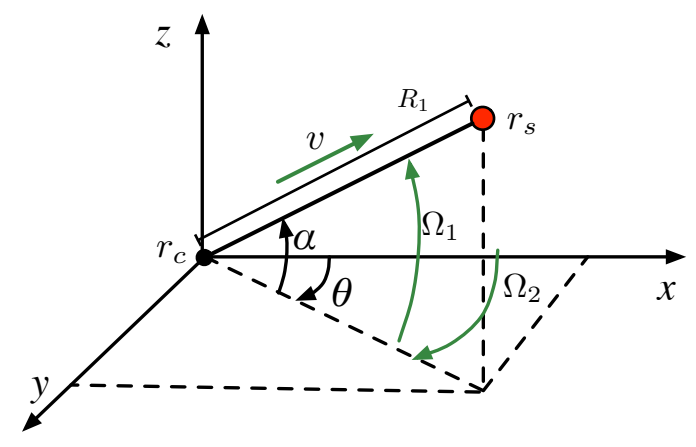

Fig. 1. Vehicle model. The vehicle sensor $r_{s}$ is a distance $R_{1}$ from the vehicle center $r_{c}$. The azimuthal angle $\alpha$ defines the pitch, whose velocity is governed by $\Omega_{1}$. The polar angle $\theta$ defines the yaw, whose velocity is governed by $\Omega_{2}$. The forward velocity, or surge velocity, is defined by $v$.

where $\frac{s}{s+h}[J]$ is a washout filter applied to the sensor reading $J$ and $a_{i}, c_{i}$ are gains affecting the control performace. As indicated, the application of extremum seeking involves both a periodic probing to search the environment, $a_{i} \omega_{i} \cos \left(\omega_{i} t\right)$ and a bias term $c_{i} \frac{s}{s+h}[J] \sin \left(\omega_{i} t\right)$ to demodulate the periodic signal and turn the vehicle in the correct direction. This allows the vehicle to converge to the source independently of position information or communication with other entities.

\section{VEHICLE MODEL}

To extend the vehicle model from a two dimensional unicycle to a three dimentional vehicle, we chose a kinematic model, depicted in Fig. 1, whose actuators can be used to impart surge, yaw and pitch velocities. The equations of motion for this vehicle are

$$
\begin{aligned}
\dot{r}_{c} & =v\left[\begin{array}{l}
\cos (\alpha) \cos (\theta) \\
\cos (\alpha) \sin (\theta) \\
\sin (\alpha)
\end{array}\right] \\
\dot{\alpha} & =\Omega_{1} \\
\dot{\theta} & =\Omega_{2}
\end{aligned}
$$

where $v$ is the surge velocity, $r_{c}$ is the center of the vehicle, and $\alpha$ and $\theta$ are the pitch and yaw angles of the vehicle respectively. The sensor is located at

$$
r_{s}=r_{c}+R\left[\begin{array}{l}
\cos (\alpha) \cos (\theta) \\
\cos (\alpha) \sin (\theta) \\
\sin (\alpha)
\end{array}\right] .
$$

To control the vehicle, we set the surge velocity to a constant and use extremum seeking to tune the angular velocities

$$
\begin{aligned}
v & =V_{c} \\
\Omega_{1} & =a \cos (\omega t)+c_{\alpha} \xi \sin (\omega t)+d_{\alpha} \xi^{2} \sin (\omega t) \\
\Omega_{2} & =-a \sin (\omega t)+c_{\theta} \xi \cos (\omega t)-d_{\theta} \xi^{2} \cos (\omega t) \\
\xi & =\frac{s}{s+h}[J]
\end{aligned}
$$

where $\omega$ is the probing frequency, the parameters $c_{\alpha}, c_{\theta}, d_{\alpha}, d_{\theta}$ affect the convergence rate, the parameter $a$ affects the accuracy of the gradient estimation, and $\frac{s}{s+h}[J]$ is a washout filter applied to the sensor reading $J$. In the next section we present a stability result for this scheme.

\section{STABILITY}

The dynamics of the closed loop are intricate. The complexity comes from the trigonometric nonlinearities in the vehicle model, the polynomial nonlinearity in the signal map, and from the time varying forcing applied by extremum seeking. The complexity of the system increases compared to the two dimensional case as two extra states must be added to account for the dynamics in the extra dimension.

We assume the nonlinear map defining the distribution of the signal field is quadratic and takes the form $J=f\left(r_{s}\right)=$ $f^{*}-q_{r}\left|r_{s}-r^{*}\right|^{2}$ where $r^{*}$ is the unknown maximizer, $f^{*}=f\left(r^{*}\right)$ is the unknown maximum and $q_{r}$ is an unknown positive constant. We define an output error variable $e=$ $\frac{h}{s+h}[J]-f^{*}$ where $\frac{h}{s+h}[J]$ is a high-pass filter applied to the sensor reading $J$, which allows us to express $\xi$, the signal from the washout filter, as $\xi=\frac{s}{s+h}[J]=J-\frac{h}{s+h}[J]=$ $J-f^{*}-e$. As a consequence $\xi$ and $\dot{e}$ take the following form

$$
\begin{aligned}
\xi & =-\left(q_{r}\left|r_{s}-r^{*}\right|^{2}+e\right) \\
\dot{e} & =h \xi
\end{aligned}
$$

Theorem 1: Consider the system defined by (2)-(8) and (10), (11) where $c_{\theta}, c_{\alpha}, d_{\theta}, d_{\alpha}, h, R, V_{c}, q_{r}>0$, the parameter $a$ is chosen such that $4 V_{c} J_{0}(\sqrt{2} a)>$ $h R\left(4 J_{0}(\sqrt{2} a)-\frac{\left(\sqrt{2} J_{1}(2 a)+J_{1}(2 \sqrt{2} a)\right)}{J_{1}(\sqrt{2} a)}\right)$ and where $J_{0}(a)$ and $J_{1}(a)$ are Bessel functions of the first kind. For sufficiently large $\omega$, if the initial conditions $r_{c}(0), \theta(0), \alpha(0), e(0)$ are such that the follow quantities are sufficiently small

$$
\begin{gathered}
|| r_{c}(0)-r^{*}|-\rho|, \quad|\alpha(0)|, \\
\left|e(0)+q_{r} R^{2}+\frac{V_{c} J_{0}(\sqrt{2} a)}{\sqrt{2} c_{\theta} R J_{1}(\sqrt{2} a)}\right| \\
\text { and either } \quad\left|\theta(0)-\arctan \frac{y_{c}-y^{*}}{x_{c}-x^{*}}+\frac{\pi}{2}\right| \\
\text { or } \quad\left|\theta(0)-\arctan \frac{y_{c}-y^{*}}{x_{c}-x^{*}}-\frac{\pi}{2}\right|
\end{gathered}
$$

where

$$
\rho=\sqrt{\frac{V_{c} J_{0}(\sqrt{2} a)}{\sqrt{2} c_{\theta} q_{r} R J_{1}(\sqrt{2} a)}},
$$

then the trajectory of the vehicle center, $r_{c}(t)$, locally exponentially converges to, and remains in, the spherical annulus

$$
\begin{gathered}
|\alpha| \leq O(1 / \omega) \\
\rho-O(1 / \omega) \leq\left|r_{c}-r^{*}\right| \leq \rho+O(1 / \omega)
\end{gathered}
$$

and the sensor reading $J(t)$ converges exponentially to a periodic function within $O(1 / \omega)$ of

$$
f^{*}-q r R^{2}-\frac{V_{c} J_{0}(\sqrt{2} a)}{\sqrt{2} c_{\theta} R J_{1}(\sqrt{2} a)} .
$$


Proof: We start the proof by defining the shifted variables

$$
\begin{aligned}
\hat{r}_{c} & =r_{c}-r^{*} \\
\hat{\alpha} & =\alpha-a \sin (\omega t) \\
\hat{\theta} & =\theta-a \cos (\omega t) \\
\tau & =\omega t
\end{aligned}
$$

and noting their dynamics

$$
\begin{aligned}
\frac{d \hat{r}_{c}}{d \tau} & =\frac{V_{c}}{\omega}\left[\begin{array}{c}
\cos (\hat{\alpha}+a \sin (\tau)) \cos (\hat{\theta}+a \cos (\tau)) \\
\cos (\hat{\alpha}+a \sin (\tau)) \sin (\hat{\theta}+a \cos (\tau)) \\
\sin (\hat{\alpha}+a \sin (\tau))
\end{array}\right] \\
\frac{d \hat{\alpha}}{d \tau}=\frac{1}{\omega}\left(c_{\alpha} \xi \sin (\tau)+d_{\alpha} \xi^{2} \sin (\tau)\right) & \frac{1}{\omega \hat{\theta}}\left(c_{\theta} \xi \cos (\tau)-d_{\theta} \xi^{2} \cos (\tau)\right) .
\end{aligned}
$$

We now redefine $r_{c}$ by its polar coordinates

$$
\begin{aligned}
\tilde{r}_{c} & =\left|\hat{r}_{c}\right|=\sqrt{\hat{x}_{c}^{2}+\hat{y}_{c}^{2}+\hat{z}_{c}^{2}} \\
\hat{r}_{c} & =\tilde{r}_{c}\left[\begin{array}{l}
\cos \left(\alpha^{*}\right) \cos \left(\theta^{*}\right) \\
\cos \left(\alpha^{*}\right) \sin \left(\theta^{*}\right) \\
\sin \left(\alpha^{*}\right)
\end{array}\right] \\
\tan \left(\theta^{*}\right) & =\frac{\hat{y}_{c}}{\hat{x}_{c}} \\
\tan \left(\alpha^{*}\right) & =\frac{\hat{z}_{c}}{\sqrt{\hat{y}_{c}^{2}+\hat{x}_{c}^{2}}} .
\end{aligned}
$$

Using these new definitions, the expression for $\xi$ is

$$
\begin{aligned}
\xi= & -q_{r}\left(\tilde{r}_{c}^{2}+R^{2}+2 \tilde{r}_{c} R \xi_{c}\right)-e \\
\xi_{c}= & \cos (\hat{\alpha}+a \sin (\tau)) \cos \left(\alpha^{*}\right) \cos \left(\hat{\theta}-\theta^{*}+a \cos (\tau)\right) \\
& +\sin (\hat{\alpha}+a \sin (\tau)) \sin \left(\alpha^{*}\right)
\end{aligned}
$$

and the resulting dynamics are

$$
\begin{aligned}
\frac{d \tilde{r}_{c}}{d \tau}= & \frac{\frac{d \hat{x}_{c}}{d \tau} \hat{x}_{c}+\frac{d \hat{y}_{c}}{d \tau} \hat{y}_{c}+\frac{d \hat{z}_{c}}{d \tau} \hat{z}_{c}}{\tilde{r}_{c}} \\
= & \frac{V_{c}}{\omega} \xi_{c} \\
\frac{d \alpha^{*}}{d \tau}= & \frac{\frac{d \hat{z}_{c}}{d \tau} \sqrt{\hat{y}_{c}^{2}+\hat{x}_{c}^{2}}-\hat{z}_{c} \frac{d \sqrt{\hat{y}_{c}^{2}+\hat{x}_{c}^{2}}}{d \tau}}{\tilde{r}_{c}^{2}} \\
= & \frac{V_{c}}{\omega}\left(\frac{\sin (\hat{\alpha}+a \sin (\tau)) \cos \left(\alpha^{*}\right)}{\tilde{r}_{c}}\right. \\
& -\frac{\cos (\hat{\alpha}+a \sin (\tau)) \sin \left(\alpha^{*}\right)}{\tilde{r}_{c}} \\
\frac{d \theta^{*}}{d \tau}= & \frac{\frac{d \hat{y}_{c}}{d \tau} \hat{x}_{c}-\hat{y}_{c} \frac{d \hat{x}_{c}}{d \tau}}{\hat{y}_{c}^{2}+\hat{x}_{c}^{2}} \\
= & \frac{V_{c}}{\omega} \frac{\cos (\hat{\alpha}+a \sin (\tau)) \sin \left(\hat{\theta}-\theta^{*}+a \cos (\tau)\right)}{\tilde{r}_{c} \cos \left(\alpha^{*}\right)} .
\end{aligned}
$$

The system order can be reduced from six to five by combining $\hat{\theta}$ and $\theta^{*}$ into the error variable

$$
\tilde{\theta}=\hat{\theta}-\theta^{*}
$$

resulting in the error system

$$
\begin{aligned}
\frac{d \tilde{r}_{c}}{d \tau}= & \frac{V_{c}}{\omega} \xi_{c} \\
\frac{d \alpha^{*}}{d \tau}= & \frac{V_{c}}{\omega}\left(\frac{\sin (\hat{\alpha}+a \sin (\tau)) \cos \left(\alpha^{*}\right)}{\tilde{r}_{c}}\right. \\
& \left.-\frac{\cos (\hat{\alpha}+a \sin (\tau)) \sin \left(\alpha^{*}\right) \cos (\tilde{\theta}+a \cos (\tau))}{\tilde{r}_{c}}\right) \\
\frac{d \hat{\alpha}}{d \tau}= & \frac{1}{\omega}\left(c_{\alpha} \xi \sin (\tau)+d_{\alpha} \xi^{2} \sin (\tau)\right) \\
\frac{d \tilde{\theta}}{d \tau}= & \frac{1}{\omega}\left(c_{\theta} \xi \cos (\tau)-d_{\theta} \xi^{2} \cos (\tau)\right) \\
& -\frac{V_{c}}{\omega} \frac{\cos (\hat{\alpha}+a \sin (\tau)) \sin (\tilde{\theta}+a \cos (\tau))}{\tilde{r}_{c} \cos \left(\alpha^{*}\right)} \\
\frac{d e}{d \tau}= & \frac{h}{\omega} \xi \\
\xi= & -q_{r}\left(\tilde{r}_{c}^{2}+R^{2}+2 \tilde{r}_{c} R \xi_{c}\right)-e \\
\xi_{c}= & \cos (\hat{\alpha}+a \sin (\tau)) \cos \left(\alpha^{*}\right) \cos (\tilde{\theta}+a \cos (\tau)) \\
& +\sin (\hat{\alpha}+a \sin (\tau)) \sin \left(\alpha^{*}\right)
\end{aligned}
$$

As the system equations are periodic in $2 \pi$, the average error system is

$$
\begin{aligned}
\frac{d \tilde{r}_{c}^{\text {ave }}}{d \tau}= & \frac{V_{c}}{\omega} \xi_{c}^{\text {ave }} \\
\frac{d \alpha^{* \text { ave }}}{d \tau}= & \frac{V_{c}}{\omega}\left(\frac{J_{0}(a) \sin \left(\hat{\alpha}^{\text {ave }}\right) \cos \left(\alpha^{* \text { ave }}\right)}{\tilde{r}_{c}^{\text {ave }}}\right. \\
& \left.-\frac{J_{0}(\sqrt{2} a) \cos \left(\hat{\alpha}^{\text {ave }}\right) \sin \left(\alpha^{* \text { ave }}\right) \cos \left(\tilde{\theta}^{\text {ave }}\right)}{\tilde{r}_{c}^{\text {ave }}}\right) \\
\frac{d \hat{\alpha}^{\text {ave }}}{d \tau}= & -\frac{2 q_{r} R \tilde{r}_{c}^{\text {ave }} \xi_{c}^{\text {ave }}}{\omega}\left(c_{\alpha}\right. \\
& \left.-2 d_{\alpha}\left(q_{r}\left(\tilde{r}_{c}^{\text {ave }}+R^{2}\right)+e^{\text {ave }}\right)\right) \\
& +\frac{4 d_{\alpha} q_{r}^{2} R^{2} \tilde{r}_{c}^{\text {ave }} \xi_{c}^{2 \text { ave }}}{\omega} \\
\frac{d \tilde{\theta}^{\text {ave }}}{d \tau}= & -\frac{2 q_{r} R \tilde{r}_{c}^{\text {ave }} \xi_{c}^{\text {ave }}}{\omega}\left(c_{\theta}\right. \\
& \left.+2 d_{\theta}\left(q_{r}\left(\tilde{r}_{c}^{\text {ave }}{ }^{2}+R^{2}\right)+e^{\text {ave }}\right)\right) \\
& -\frac{4 d_{\theta} q_{r}^{2} R^{2} \tilde{r}_{c}^{\text {ave }} \xi_{c}^{2 \text { ave }}}{\omega} \\
& -J_{0}(\sqrt{2} a) \frac{V_{c}}{\omega} \frac{\cos \left(\hat{\alpha}^{\text {ave }}\right) \sin \left(\tilde{\theta}^{\text {ave }}\right)}{\tilde{r}_{c}^{\text {ave }} \cos \left(\alpha^{* a v e}\right)} \\
&
\end{aligned}
$$

$\frac{d e^{\text {ave }}}{d \tau}=-\frac{h}{\omega}\left(\left(q_{r}\left(\tilde{r}_{c}^{\text {ave }^{2}}+R^{2}\right)+e\right)+2 q_{r} R \tilde{r}_{c}^{\text {ave }} \xi_{c}^{\text {ave }}\right)(50)$ 
where

$$
\begin{aligned}
& \xi_{c}^{\text {ave }}=J_{0}(\sqrt{2} a) \cos \left(\alpha^{*^{\text {ave }}}\right) \cos \left(\hat{\alpha}^{\text {ave }}\right) \cos \left(\tilde{\theta}^{\text {ave }}\right) \\
& +J_{0}(a) \sin \left(\alpha^{*^{\text {ave }}}\right) \sin \left(\hat{\alpha}^{\text {ave }}\right) \\
& \xi_{c}^{\sin }=-\frac{J_{1}(\sqrt{2} a)}{\sqrt{2}} \cos \left(\alpha^{*^{\text {ave }}}\right) \sin \left(\hat{\alpha}^{\text {ave }}\right) \cos \left(\tilde{\theta}^{\text {ave }}\right) \\
& +J_{1}(a) \sin \left(\alpha^{*^{\text {ave }}}\right) \cos \left(\hat{\alpha}^{\text {ave }}\right) \\
& \xi_{c}^{\text {ave }}=-\frac{J_{1}(\sqrt{2} a)}{\sqrt{2}} \cos \left(\alpha^{*^{\text {ave }}}\right) \cos \left(\hat{\alpha}^{\text {ave }}\right) \sin \left(\tilde{\theta}^{\text {ave }}\right) \\
& \xi_{c}^{\text {ain }}=-\frac{\cos ^{2}\left(\alpha^{*^{\text {ave }}}\right)}{4}\left(J_{1}(2 a) \sin \left(2 \hat{\alpha}^{\text {ave }}\right)\right. \\
& \left.+\frac{J_{1}(2 \sqrt{2} a)}{\sqrt{2}} \sin \left(2 \hat{\alpha}^{\text {ave }}\right) \cos \left(2 \tilde{\theta}^{\text {ave }}\right)\right) \\
& +J_{1}(2 a) \frac{\sin ^{2}\left(\alpha^{*^{\text {ave }}}\right)}{2} \sin \left(2 \hat{\alpha}^{\text {ave }}\right) \\
& +2 \frac{J_{1}(\sqrt{5} a)}{\sqrt{5}} \frac{\sin \left(2 \alpha^{*^{\text {ave }}}\right)}{2} \cos \left(2 \hat{\alpha}^{\text {ave }}\right) \cos \left(\tilde{\theta}^{\text {ave }}\right)(54) \\
& \xi_{c}^{2^{\text {ave }}}=-\frac{\cos ^{2}\left(\alpha^{*^{\text {ave }}}\right)}{4}\left(J_{1}(2 a) \sin \left(2 \tilde{\theta}^{\text {ave }}\right)\right. \\
& \left.+\frac{J_{1}(2 \sqrt{2} a)}{\sqrt{2}} \cos \left(2 \hat{\alpha}^{\text {ave }}\right) \sin \left(2 \tilde{\theta}^{\text {ave }}\right)\right) \\
& -\frac{J_{1}(\sqrt{5} a)}{\sqrt{5}} \frac{\sin \left(2 \alpha^{*^{\text {ave }}}\right)}{2} \sin \left(2 \hat{\alpha}^{\text {ave }}\right) \sin \left(\tilde{\theta}^{\text {ave }}\right) \text {. }
\end{aligned}
$$

The average system (46)-(50) has two equilibria defined by

$$
\begin{aligned}
& {\left[\tilde{r}_{c}^{\mathrm{ave}^{\mathrm{eq}}{ }_{1}}, \alpha^{*^{\mathrm{ave}^{\mathrm{eq}} \mathrm{q}_{1}}}, \hat{\alpha}^{\mathrm{ave}^{\mathrm{eq}{ }_{1}}}, \tilde{\theta}^{\mathrm{ave}^{\mathrm{eq}}{ }_{1}}, e^{\mathrm{ave}^{\mathrm{eq}_{1}}}\right]} \\
& =\left[\rho, 0,0, \frac{\pi}{2},-q_{r}\left(\rho^{2}+R^{2}\right)\right] \\
& {\left[\tilde{r}_{c}^{\mathrm{ave}^{\mathrm{eq}_{2}}}, \alpha^{*^{\mathrm{ave}^{\mathrm{eq}} \mathrm{q}_{2}}}, \hat{\alpha}^{\mathrm{ave}^{\mathrm{eq}_{2}}}, \tilde{\theta}^{\mathrm{ave}^{\mathrm{eq}_{2}}}, e^{\mathrm{ave}^{\mathrm{eq}_{2}}}\right]} \\
& =\left[\rho, 0,0,-\frac{\pi}{2},-q_{r}\left(\rho^{2}+R^{2}\right)\right] \text {. }
\end{aligned}
$$

These equilibria have the corresponding Jacobians

$$
\begin{aligned}
A^{\mathrm{eq}_{1}}= & {\left[\begin{array}{ccccc}
0 & 0 & 0 & -m_{14} & 0 \\
0 & -m_{22} & -m_{23} & 0 & 0 \\
0 & m_{32} & 0 & 0 & 0 \\
m_{41} & 0 & 0 & -m_{44} & m_{45} \\
-m_{51} & 0 & 0 & m_{54} & -h
\end{array}\right] } \\
A^{\mathrm{eq}_{2}}= & {\left[\begin{array}{ccccc}
0 & 0 & 0 & m_{14} & 0 \\
0 & -m_{22} & m_{23} & 0 & 0 \\
0 & -m_{32} & 0 & 0 & 0 \\
-m_{41} & 0 & 0 & -m_{44} & -m_{45} \\
-m_{51} & 0 & 0 & -m_{54} & -h
\end{array}\right] }
\end{aligned}
$$

where

$$
\begin{aligned}
m_{14}= & V_{c} J_{0}(\sqrt{2} a) \\
m_{22}= & \frac{d_{\alpha} q_{r} R V_{c} J_{0}(\sqrt{2} a)}{c_{\theta} J_{1}(\sqrt{2} a)} \\
& \times\left(\sqrt{2} J_{1}(2 a)-J_{1}(2 \sqrt{2} a)\right) \\
m_{23}= & 2 c_{\alpha} q_{r} R \rho J_{1}(a) \\
m_{32}= & V_{c} J_{0}(a) \frac{\sqrt{\sqrt{2}}}{\rho} \\
m_{41}= & m_{41 a}+m_{41 b} \\
m_{41 a}= & 4 c_{\theta} q_{r} R \frac{J_{1}(\sqrt{2} a)}{\sqrt{2}} \\
m_{41 b}= & 4 \frac{d_{\theta} q_{r} V_{c} J_{0}(\sqrt{2} a)}{c_{\theta}} \\
m_{44}= & \frac{d_{\theta} q_{r} R V_{c} J_{0}(\sqrt{2} a)}{c_{\theta} J_{1}(\sqrt{2} a)} \\
& \times\left(\sqrt{2} J_{1}(2 a)+J_{1}(2 \sqrt{2} a)\right) \\
m_{45}= & 4 d_{\theta} q_{r} R \rho \frac{J_{1}(\sqrt{2} a)}{\sqrt{2}} \\
m_{54}= & 2 h q_{r} \rho \\
= & 2 h q_{r} R \rho J_{0}(\sqrt{2} a) \\
= &
\end{aligned}
$$

The characteristic polynomial for both equilibria is

$$
\begin{aligned}
0= & \left(s^{2}+m_{22} s+m_{32} m_{23}\right) \\
& \times\left(s^{3}+\left(h+m_{44}\right) s^{2}\right. \\
& \left.+\left(h m_{44}+m_{41} m_{14}-m_{54} m_{45}\right) s+h m_{14} m_{41 a}\right)
\end{aligned}
$$

The second order polynomial has roots with negative real parts as both $m_{22}$ and $m_{32} m_{23}$ are positive. The third order polynomial has roots with negative real parts as, according to the assumptions in Theorem 1, all the coefficients are positive and the product of the $s^{2}$ and $s^{1}$ coefficients is greater than the $s^{0}$ coefficient. Therefore, the Jacobians (58), (59) are Hurwitz given the assumptions in Theorem 1. As such, both equilibria (56), (57) are exponentially stable. By applying Theorem 10.4 from [17] to this result, we conclude that the error system (46)-(50) has two distinct, exponentially stable periodic solutions within $O(1 / \omega)$ of the equilibria (56), (57). This indicates the angle $\alpha$ remains within $O(1 / \omega)$ and the distance between the vehicle center $r_{c}$ and the source $r^{*}$ converges to within $O(1 / \omega)$ of the value $\rho=\sqrt{\frac{V_{c} J_{0}(\sqrt{2} a)}{\sqrt{2} c_{\theta} q_{r} R J_{1}(\sqrt{2} a)}}$, defining the attractor in Theorem 1. As the attractive solution of $e$ is a periodic function within $O(1 / \omega)$ of $-q_{r} R^{2}-\frac{V_{c} J_{0}(\sqrt{2} a)}{\sqrt{2} c_{\theta} R J_{1}(\sqrt{2} a)}$, the sensor reading $J(t)$ converges to a periodic function within $O(1 / \omega)$ of $f^{*}-q_{r} R^{2}-\frac{V_{c} J_{0}(\sqrt{2} a)}{\sqrt{2} c_{\theta} R J_{1}(\sqrt{2} a)}$.

Remark 1: The attractor seen in this $3 \mathrm{D}$ scenario is very similar to the attractor seen in the 2D unicycle with a constant forward velocity and tuned angular velocity. In both cases the vehicle moves around an "orbit" confined to a plane - 


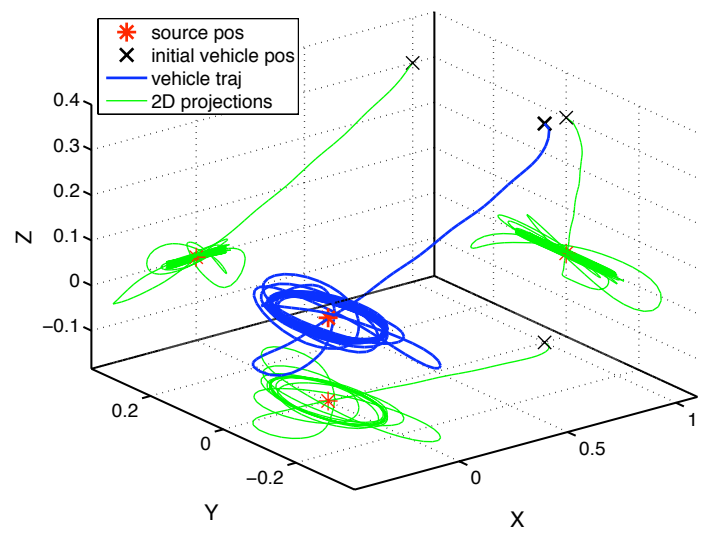

Fig. 2. Vehicle locating a static source which creates a signal field with spherical level sets. $V_{c}=.1, r=.1, a=.5, \omega=40, c_{\theta}=c_{\alpha}=$ $100, d_{\theta}=d_{\alpha}=300$

though in the 3D case the plane in question has an $O(1 / \omega)$ thickness.

\section{ILlustration of Vehicle Behavior}

The behavior shown by the vehicle is very interesting in terms of how it changes with the chosen parameters. We start this section by illustrating the behavior predicted by Theorem 1. We then examine what happens when we change the parameters in ways not covered by the theory.

The following figures illustrate the behavior predicted defined in (16)-(17). Fig. 2 shows the vehicle starting from different positions and orientations converging to an "orbit" around a static source which produces a signal field with spherical level sets. Fig. 3 illustrates the different attractors seen when the parameters are varied within the assumptions of Theorem 1. Fig. 4 shows the vehicle converging to attractors around a static source which produces a signal field with ellipsoidal level sets. Though the theory presented here does not include ellipsoidal level sets, the convergence to the attractor in these cases is similar to the convergence seen in the 2D cases where the target signal field is made up of elliptical level set. The control law (7)-(8) also allows the vehicle to seek a moving source as seen in Fig. 5 where the source follows a saddle pattern and produces spherical level sets which move with the source.

The proof of Theorem 1 relies on both $d_{\alpha}$ and $d_{\theta}$ being positive, however, convergent behavior is still seen when both are negative and when $d_{\alpha}$ is made negative. The fourth combination, when $d_{\theta}$ is negative and $d_{\alpha}$ is positive results in unstable behavior. Fig. 6 illustrates the convergent behavior when both $d_{\alpha}$ and $d_{\theta}$ are negative. In this case the attractor seen when both parameters are positive rotates and is twisted slightly. The attractor in this case is still similar to an "orbit". This differs from the third case, illustrated in Fig. 7 where the attractor is no longer of an "orbit" type. In this case the vehicle moves around the surface of a sphere maintaining $O(1 / \omega)$ within a specific distance from the source. The unstable fourth case is shown in Fig. 8.

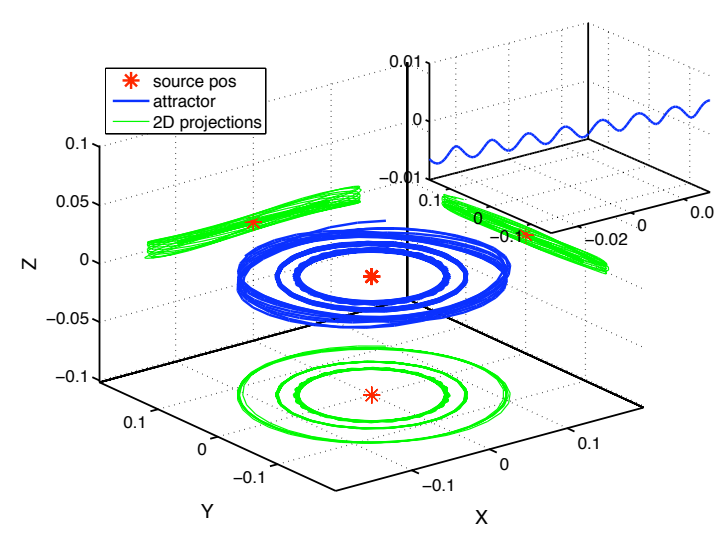

Fig. 3. Attractors resulting from different parameter configurations. The inset reveals the close-up behavior of the vehicle center. $V_{c}=.1, r=$ $.1, a=.5, \omega=40$ outer attractor: $c_{\theta}=c_{\alpha}=100, d_{\theta}=d_{\alpha}=300$ middle attractor: $c_{\theta}=200, c_{\alpha}=100, d_{\theta}=600, d_{\alpha}=300$ inner attractor: $c_{\theta}=300, c_{\alpha}=100, d_{\theta}=600, d_{\alpha}=300$



Fig. 4. Vehicle locating a target from a signal field with ellipsoidal level sets. The attractor seen has elements similar to the attractors seen in the 2D case. $V_{c}=.1, r=.1, a=.5, \omega=40, c_{\theta}=c_{\alpha}=100, d_{\theta}=300, d_{\alpha}=$ 200

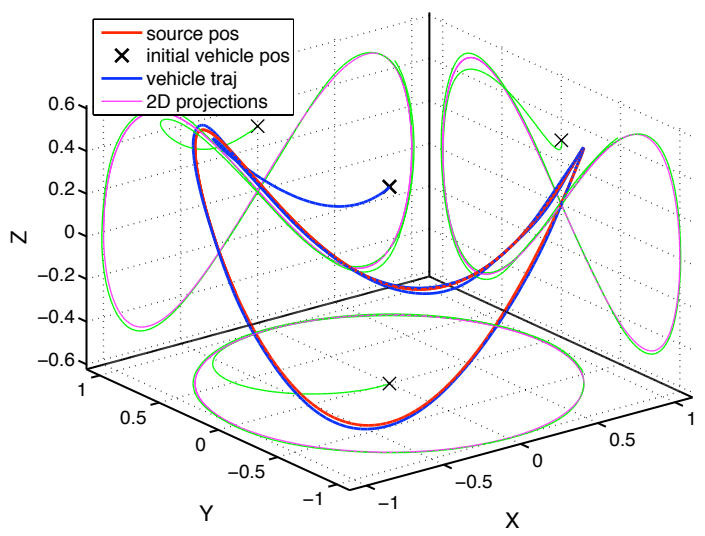

Fig. 5. The vehicle follows the moving source which creates a signal field with spherical level sets which move with the target. $V_{c}=.07, r=.1, a=$ $.5, \omega=10, c_{\theta}=c_{\alpha}=100, d_{\theta}=d_{\alpha}=300$ 


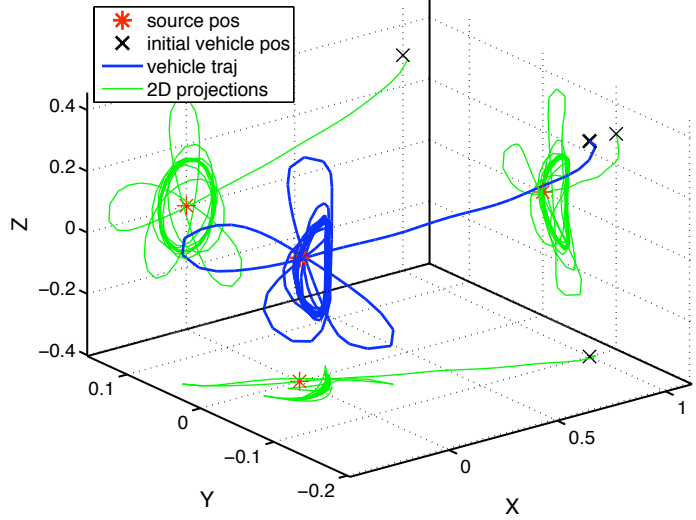

Fig. 6. Vehicle locates a source. Signal field has spherical level sets. The final attractor is rotated compared to other cases, but is still of an "orbitlike" form. $V_{c}=.1, r=.1, a=.5, \omega=40, c_{\theta}=c_{\alpha}=100, d_{\theta}=$ $300,=d_{\alpha}=-300$

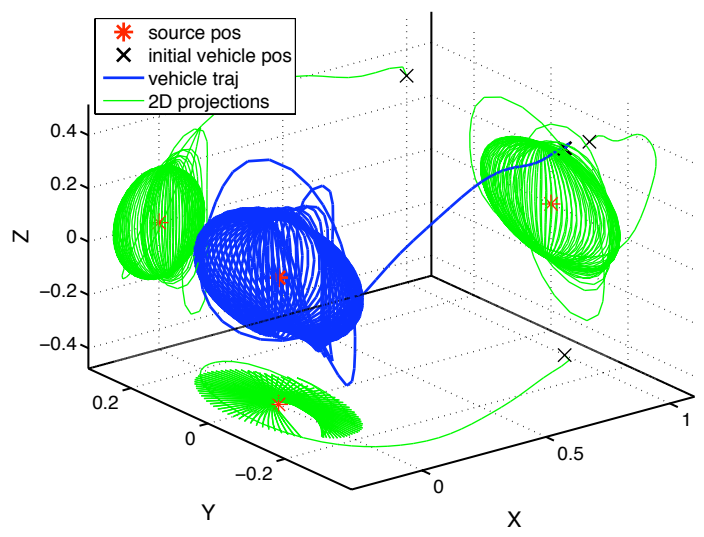

Fig. 7. Vehicle locates a source. Signal field has spherical level sets. The attractor is $O(1 . \omega)$ within the surface of a sphere instead of an "orbit" type. $V_{c}=.2, r=.1, a=.5, \omega=40, c_{\theta}=c_{\alpha}=100, d_{\theta}=300, d_{\alpha}=-300$

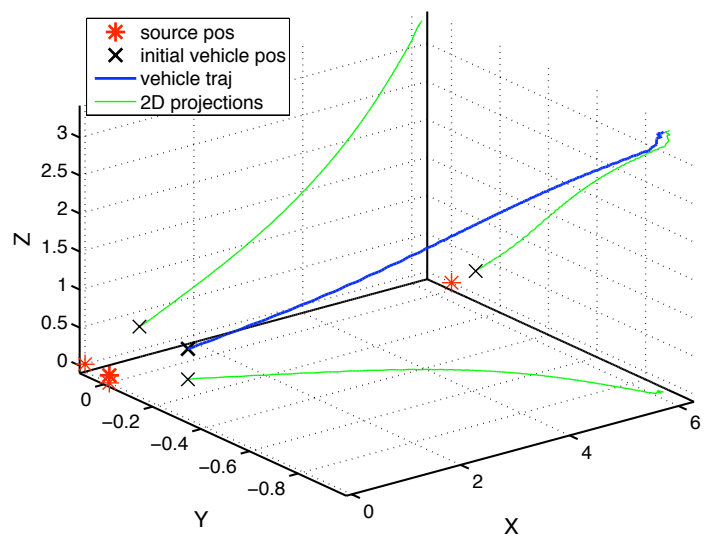

Fig. 8. Vehicle does not locate the source - parameters produce an unstable result. Signal field has spherical level sets. $V_{c}=.1, r=.1, a=.5, \omega=$ $40, c_{\theta}=c_{\alpha}=100, d_{\theta}=-300, d_{\alpha}=300$

\section{CONCLUSIONS}

We extended previous source seeking results from a two dimensional unicycle to a three dimensional underactuated vehicle and proved local exponential convergence to an attractor around the source. The vehicle behavior was illustrated through several simulations depicting different scenarios.

\section{REFERENCES}

[1] J. Cochran and M. Krstic, "Source seeking with a nonholonomic unicycle without position measurements and with tuning of angular velocity — part I: Stability analysis," 46th IEEE Conf. on Decision and Control, 2007.

[2] C. Zhang, D. Arnold, N. Ghods, A. Siranosian, and M. Krstic, "Source seeking with nonholonomic unicycle without position measurement and with tuning of forward velocity," Systems and Control Letters, vol. 56, pp. 245-252, 2007.

[3] B. Porat and A. Neohorai, "Localizing vapor-emitting sources by moving sensors," IEEE Trans. Signal Processing, vol. 44, pp. 10181021, 1996.

[4] P. Ogren, E. Fiorelli, and N. Leonard, "Cooperative control of mobile sensor networks: adaptive gradient climbing in a distributed environment," IEEE Trans. Automat. Contr., vol. 29, pp. 1292-1302, 2004.

[5] E. W. Justh and P. S. Krishnaprassad, "Natural frames and interacting particles in three dimensions," 44th IEEE Conf. on Decision and Ctrl and European Ctrl. Conf., 2005.

[6] D. J. Klein, C. Matlack, and K. A. Morgansen, "Cooperative target tracking using oscillator models in three dimensions," 2007 American Ctrl. Conf., 2007.

[7] K. Peterson and A. Stefanopoulou, "Extremum seeking control for soft landing of and electromechanical valve actuator," Automatica, vol. 29, pp. 1063-1069, 2004.

[8] Y. Ou, C. Xu, E. Schuster, T. Luce, J. R. Ferron, and M. Walker, "Extremum-seeking finite-time optimal control of plasma current profile at the diii-d tokamak," 2007 American Ctrl. Conf., 2007.

[9] C. Centioli, F. Iannone, G. Mazza, M. Panella, L. Pangione, S. Podda, A. Tuccillo, V. Vitale, and L. Zaccarian, "Extremum seeking applied to the plasma control system of the Frascati Tokamak Upgrade," 44th IEEE Conf. on Decision and Ctrl., and the European Ctrl. Conf., 2005.

[10] Y. Tan, D. Nesic, and I. M. Y. Mareels, "On non-local stability properties of extremum seeking controllers," Automatica, vol. 42, pp. 889-903, 2006.

[11] R. King, R. Becker, G. Feuerbach, L. Henning, R. Petz, W. Nitsche, O. Lemke, and W. Neise, "Adaptive flow control using slope seeking," 14th IEEE Mediterranean Conf. on Ctrl. Automation, 2006.

[12] R. Becker, R. King, R. Petz, and W. Nitsche, "Adaptive closedloop separation control on a high-lift configuration using extremum seeking," 3rd AIAA Flow Control Conference, 2006.

[13] M. Tanelli, A. Astolfi, and S. Savaresi, "Non-local extremum seeking control for active braking control systems," Conference on Control Applications, 2006

[14] M. Guay, M. Perrier, and D. Dochain, "Adaptive extremum seeking control of nonisothermal continuous stirred reactors," Chem. Eng. Science, vol. 60, pp. 3671-3681, 2005.

[15] Y. Li, A. Rotea, G. T.-C. Chiu, L. Mongeau, and I.-S. Paek, "Extremum seeking control of a tunable thermoacoustic cooler," IEEE Trans. Contr. Syst. Technol., vol. 13, pp. 527-536, 2005.

[16] X. Zhang, D. Dawson, W. Dixon, and B. Xian, "Extremum seeking nonlinear controllers for a human exercise machine," in Proc. 2004 IEEE Conf. Decision and Ctrl., 2004.

[17] H. Khalil, Nonlinear Systems, 3rd ed. Upper Saddle Rive, NJ: Prentice Hall, 2002 